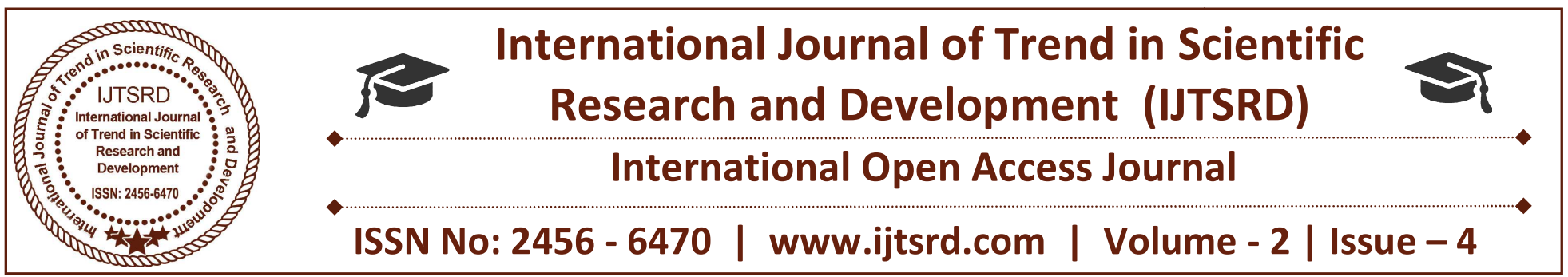

\title{
Fruit Farm Surveillance Using Drones
}

\author{
Adarsh $A^{1}$, Pranav $P M^{2}$, Manjunath $C R^{3}$, Soumya $K N^{4}$ \\ ${ }^{1,2}$ B.Tech Final Year, ${ }^{3}$ Associate Professor, ${ }^{4}$ Assistant Professor \\ ${ }^{1,2,4}$ Department of Information Science \& Engineering, \\ ${ }^{3}$ Department of Computer Science and Engineering, \\ ${ }^{1,2,3,4}$ School of Engineering \& Technology - Jain University, Bangalore, Karnataka, India
}

\section{ABSTRACT}

Monitoring all the farm activities manually takes a lot of time and is not efficient. In the recent days farmers spent a lot of time in providing quality results, but it is not $100 \%$ accurate. In other countries, the plantation area is much larger in size compared to India. In order to monitor, they've to hire more workers. Instead when the whole land is divided into parts, a drone can monitor each part by traversing from one end to the other. And the drone can be controlled by a single person sitting at any corner of the world. The drones have high resolution camera's fixed on it, which can scan the entire land for accurate results. These highresolution cameras can detect the percentage of fruitful plants and which aren't. Once this is detected, farmers can find alternate ways for the best results. It's hard to control the large plantation area manually, hence we use drones. To overcome this, drones can be used to find the percentage of fruitful/infected plants using image processing. It's easier to find the percentile of plants which will provide fruits in the near future. This also helps to cover large areas, reduce manual work, and can be used to extract the required results. Drones can be used to avoid bird feasting in the plantation area.

Keywords: Ripeness Detection, Drone Technology in Agriculture, Image Processing for Ripeness Detection, Crop Surveillance.

\section{INTRODUCTION}

Drones are being used commercially for agricultural purpose from many years, to be precise drones are used since early 1980 's. They are usually an aerial robot which would either be in charge of a person via audio commands/wireless controllers sitting at a corner or it could be made autonomous(preprogrammed) as well. This vast technology in cultivation is being used for different applications including spraying pesticides on crops, monitoring weeds, pests, and nutritional deficiencies if any. The use of advanced sensors and imaging features on drones are used to enhance a richer representation of the plantation area.

Now, the agricultural drones can be used in large plantation which can totally minimize a huge financial loss faced by the farmers. Drones that are used in agricultural sectors are termed as "Agricultural Drones". This type of drones is the one which can be implemented to farming that can help to increase crop yield rate, monitor crop healthiness and it can also help farmers to monitor the crop growth periodically which saves labour work.

By the use of these stepped forward sensors and other capabilities that are established on drones, grazers (farmers) may be able to use such drones to assist them huddle a richer exact likeness of their fields as voiced inside the introductory wording. Data that's human collected through such device might prove useful in improving crop health and graze adaptability. Such drones (Agricultural drones) favour farmers to see their plantation area (farm) from a 
greater height (say sky). These abilities not only decrease manual work, but also decreases the budget which has to be spent for labour work and provide accurate results.

When plantation area is being captured from sky, such view helps to tackle many major issues like pest or fungal infestations, crop health, and soil variation etc....

Infrared as well as visual spectrum view can be seen through multispectral images that are been captured by drones. Such combination helps the farmers to categorize healthier and infected plants which later helps them to take pression measures to avoid the disease to be spread from infected plant life to the enclosed healthier vegetation. The difference between affected and unaffected plants can't be seen through a human eye, so having the facility to view the farm area with multispectral images, it is helping the farmer with assessing crop growth as well as crop yielding.

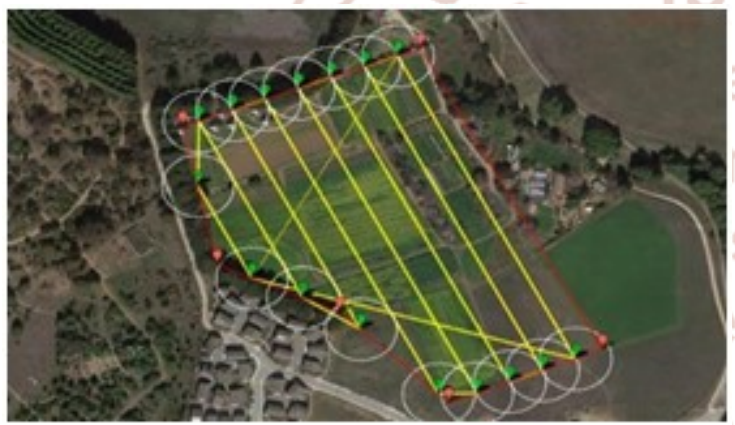

Figure 1.1: Area detection using drones

Also, farmer can run drone whenever he wants to, crops can be monitored every month/week/even everyday as per the owner's liking. Even seasonal changes could be seen over drones that makes it well known in crop inspection (Crop surveillance). These surveys can show changes in the field over time, thus helping the farmer to highlight the trouble spots.

This is definitely the prime advantage of drones in crop monitoring because by knowing the trouble spots, farmers can plan their easy methods to recover overall production of their crops. In the existing situation, drones are likewise being used for surveillance, traffic monitoring, weather monitoring etc.

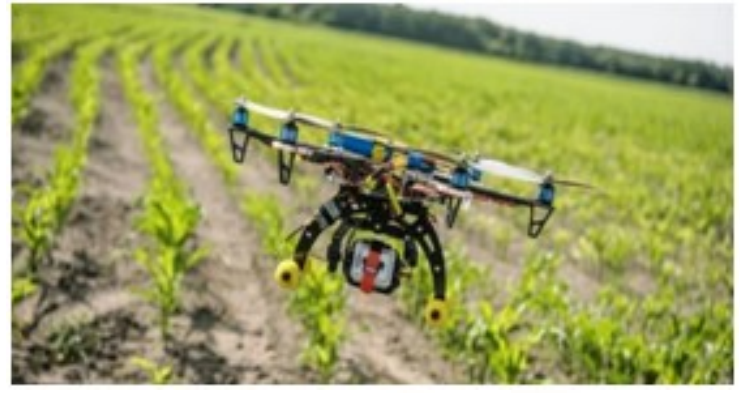

Figure 1.2: Crop surveillance

In this paper, we concentrate on how drones can be used to determine the number of fruitful mango plants which makes the farmer's life easy and saves huge money which has to be spent for manual work. The shape, size, and color of mangoes determine which plant is fruitful and which is defected. The defective plants may be treated at the earlier stages which might prevent a huge loss. As quoted earlier the estate can be monitored whenever the producer wishes to.

\section{Existing System}

The different strategies in agriculture utilizing drone technology are:

\section{Planting Seeds:}

With current progressions in ramble innovation, they've possessed the capacity to diminish seed planting costs fundamentally. The way this works is that automatons would fly $300+$ feet over their assigned zones to gather data on the site. Utilizing this data, these automatons would drop "seed cases" in regions that their seeds are destined to prosper. This technique for seed planting is right around 10 times quicker than people planting trees by hand and can conceivably diminish general expenses considerably. Using automatons to start new products is particularly advantageous in areas where it may be especially troublesome for agriculturists to plant seeds in. Amid amazingly hot circumstances of the year, utilizing automatons would be an awesome other option to having to physically plant them.

\section{Spraying Pesticides:}

By utilizing a progression of various separation measure/imaging hardware like LiDAR, drones can viably evade obstructions amid their flights. At first, the way UAVs would explore their environment would be through remote controls that should have been explored physically. I.e., if the automaton pilot wasn't cautious, the automaton could without much of a stretch keep running into a tree and crash. Be that as 
it may, with new innovation, this is effectively avoidable.

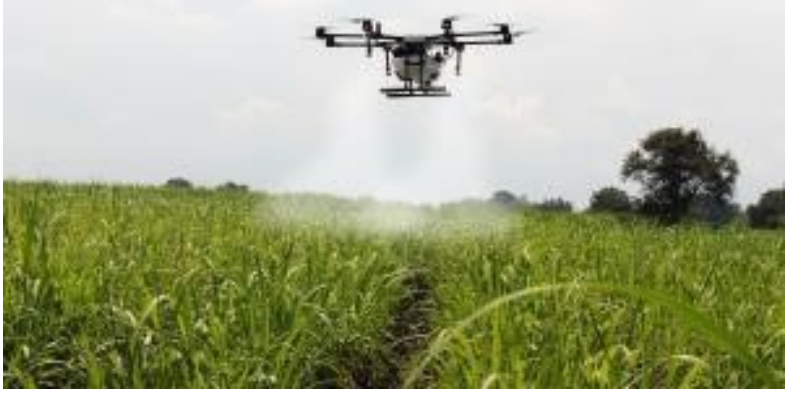

Figure 2.2: Spraying pesticides

Instruments like sensors that utilize ultrasonic resounding and LiDAR enable drones to abstain from running into snags around them amid flight. All things considered, it's presently attainable for automatons to fly at sufficiently low heights to splash pesticides and target specific segments of a field to appropriate it in likely manner. By utilizing drones as opposed to pesticide-splashing planes, ranchers would now be able to target precisely which crops require pesticides and how much should be showered while the automatons really splash them.

\section{Overall Analysis of Crop Fields:}

Agribusiness is one industry where the significance of Big-Data isn't underlined enough. Utilizing hyperspectral imaging innovation, agriculturists can accomplish unimaginably valuable data that would enable them to expand their tasks. Contingent upon the imaging technique, automatons can enable ranchers to do the accompanying as appeared in the figure underneath.

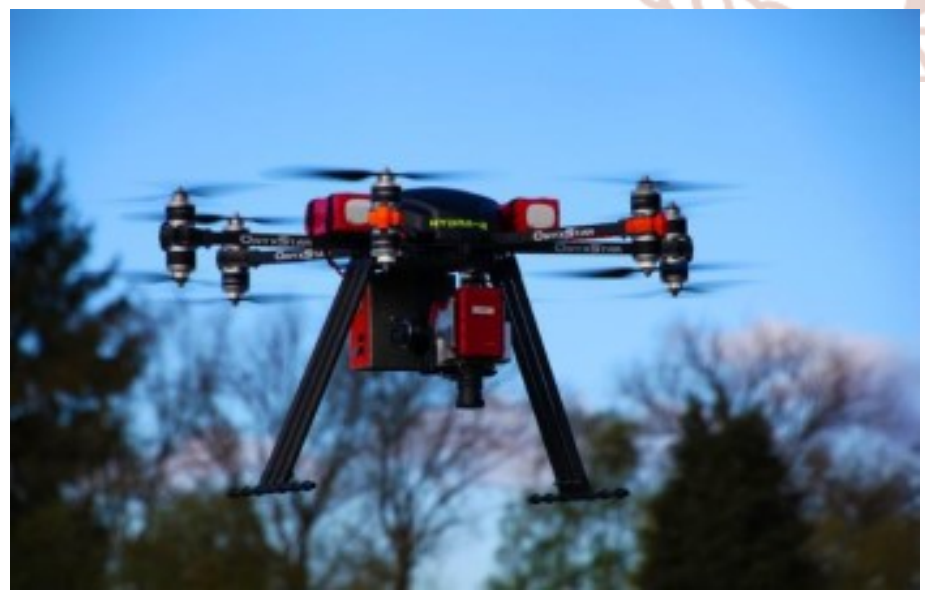

Figure 2.3: Monitoring the field using drones
It evaluates crop's well-being, spot contagious fungal defilement on trees, find maturing bottlenecks, find poor water system, and accumulate general data on environmental conditions. With such an assorted scope of noteworthy data accessible at an agriculturist's disposal, they can settle on educated choices in view of solid information. As opposed to making surmises on what the scene will look like later on, ranchers would rather have the capacity to make prevision.

\section{Methodologies in farm monitoring}

\section{DRONES:}

Drones are used for several purposes. In agricultural industry these drones will make a huge difference with planning and strategy based on the real-time data processing. As said earlier, drones have many advantages depending on the type and cause. Aerial and ground-based drones will be used in the entire crop cycle:

\section{Surveillance:}

Agricultural drones let farmers see their fields from a greater height, say (sky). This bird's-eye view can reveal many defects in plantation area like irrigation problems, soil variation, and of course, pest and fungal infestation. Multispectral images captured through the drone's project a near-infrared sight plus a visual spectrum analysis. The combination of these images shows the producer the variations enclosed by lively and infected plants. This difference isn't always precisely detectable to the human eye, so having the ability to see the crops from the abovementioned views might help the labourer with assessing crop growth, in addition crop production.

Additionally, the drone can overlook the crops for the producer regularly to their preference. Weekly, each day, or perhaps hourly, pictures can exhibit the changes within the crops over time, thus projecting possible "trouble spots". This proves to be a key benefit because by identifying the particular trouble spots, the grower can then try to recover crop management and recuperate the final yielding of their crop.

\section{Monitoring:}

Large fields with low efficiency in crop monitoring in combination build farming's greatest disincentive. Monitoring demanding situations are exacerbated by 
many uncertain climatic conditions, that steer endanger and field preservation costs. Previously, satellite imagery offered the main stepped forward form of monitoring. But there were drawbacks. Images had to be ordered in advance, could be taken only once every day, and were imprecise. Further, services were exceptionally valuable and the pictures' high quality in general suffered on various days.

Today, time-series animations can show the precise development of a crop and reveal production inefficiencies, enabling better crop management.

\section{Spraying Pesticides:}

Spraying insecticide with the help of aerial vehicles will be the leading-edge approach utilized in the sector of agriculture. Drone automation primarily based unmanned aerial vehicle (UAV) has the flexibility for smooth scouting over crop fields, collecting exact message and transmitting the information on a problem-solving time principle. This effectiveness could be passed down for the benefit of one's grazing region at sectional, resident adjust for assessing land and crop strength; extent, sample and harshness of injury along with issuing forewarning, post-event supervision and establishment of payment under crop safeguard schemes. Drones are used to spray fungicide in order to avoid the spread of plant diseases. These drones allow the farmers to establish the amount of fruits which can be plucked from each tree. There's also another advantage where farmers might know the precise spots where the fruits are ready for harvest and the count of fruits in each tree. This feature will decrease the time of farmers and highly improve the efficiency and accuracy in harvesting. Now, these drones can be widely used in any horticulture and floriculture sectors as mentioned previously. Drones lend a hand to monitor the diseases of larger trees like mango farms using high definition cameras installed it, where, it is challenging for humans to climb the tree. The drones can effortlessly sprinkle pesticides on the particular trees. Drones can monitor the crop quality with thermal sensors. By integrating improved image or video processing units to the drones, they are able to perform miracles in agro-industry.

\section{IMAGE PROCESSING:}

\section{Shape and Texture:}

Shape in the sense graphical info which contains the appropriate location, size and rotational effects are filtered out. Grading applied to many fruits and vegetables. An image texture is usually a set of attributes determined in image processing designed in finding the pattern of an image. Image texture/pattern gives us information regarding the image colour or intensity. Image textures are one of the ways which can be utilized to assist in segmentation or classification of images. To analyse an image texture in computer graphics, there are actually two methods to approach the problem: Structured Approach and Statistical Approach. In this one, the evaluation is made on how the several parameters may be used for automatic fruit grading system.

\section{Colour and Ripeness:}

We are illustrating the several approaches to discover the rate of ripeness(evolution) of fruits. Here, we use approaches like histogram matching algorithms, clustering methods primarily based image separation and the relative value of parameter-based segmentation etc. Each technique uses coloured images of fruits as input data.

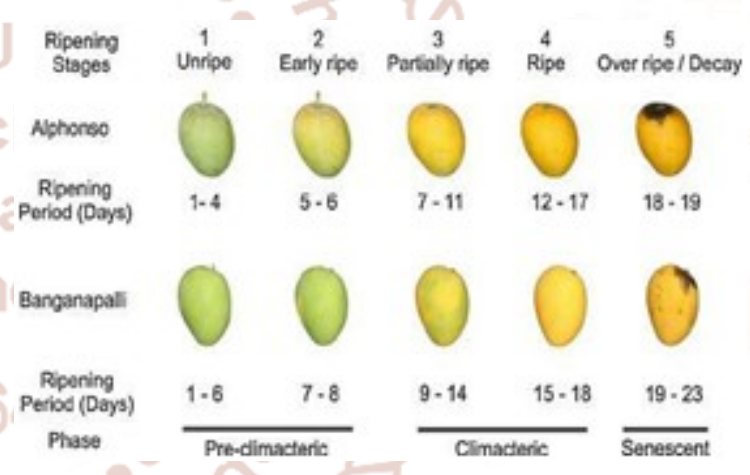

Figure 3.2: Stages of ripeness in mangoes

In these techniques, we set some threshold levels. By comparing the input(hard-coded) data image with the particular threshold level, we are able to find the maturity level of provided fruits.

\section{GPS TRACKING SYSTEM:}

\section{Mixed farming:}

We can embed GPS tracker at the trackers or signalling device to discover the bounds of your specific plantation and to maintain and monitor that area.

\section{Large trees:}

We can implant GPS system for every tree so that drones can track every tree specifically. This helps to 
increase the accuracy in processing, maintaining, and monitoring of fruit and tree's health.
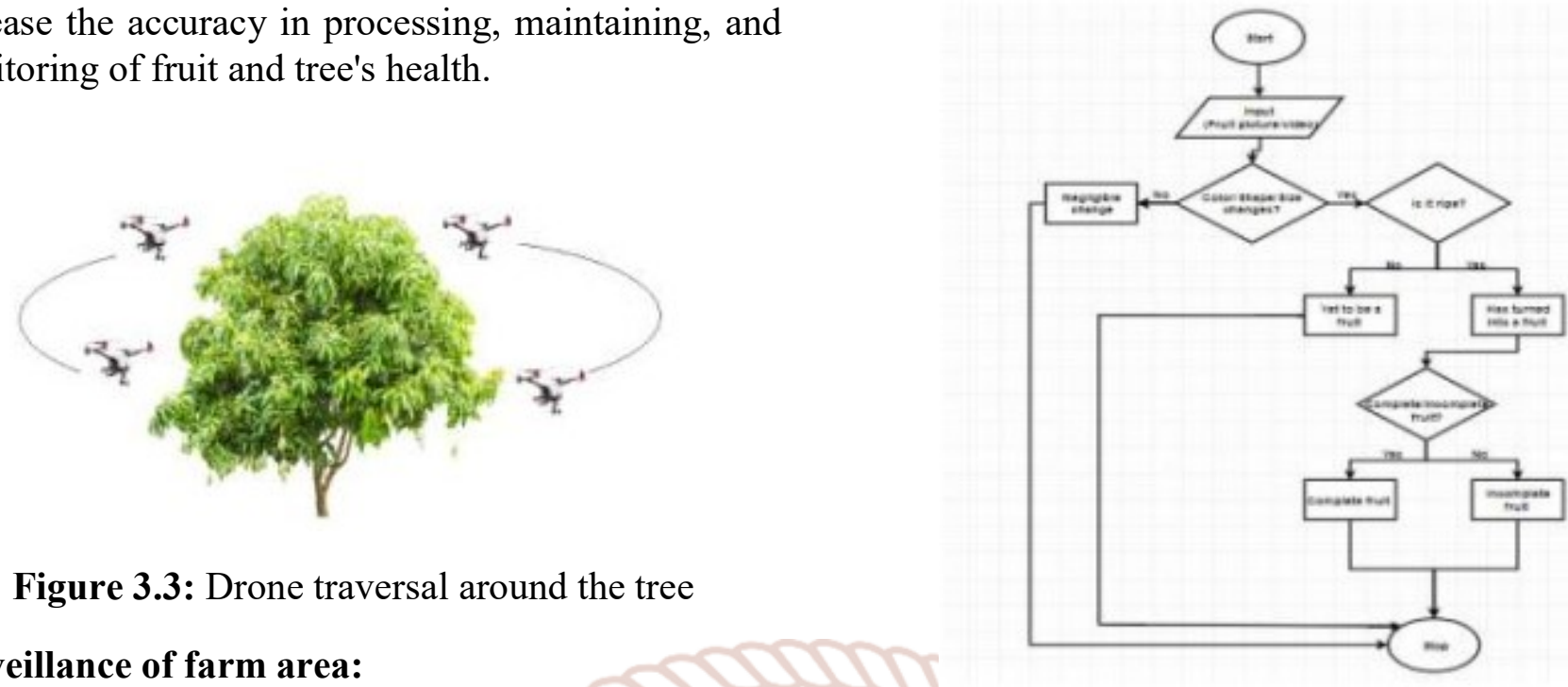

Figure 3.3: Drone traversal around the tree

\section{Surveillance of farm area:}

For complete farm area, each plant can be monitored by pre-installing a signalling device on the borderline of the plantation area so that the drone will travel in the described line consistent with the grazer's need. This also helps the farmer to change the coordinates so that the drone can examine the entire farm land.

\section{Proposed System}

The existing system (Crop surveillance using drones) can be improvised by adding new featuring methods similar to fruit growth detection, fruit health monitoring. This feature is applicable for crops such as mango trees, banana plants, orange trees, apple trees, etc... These changes can be analysed by image processing algorithms like Clustering algorithms, Histogram matching, and Parameter based segmentation. Farmers can set a threshold value and apply image processing algorithms. Changes in the fruit colour can be observed, and if the plant is infected, it can be treated or removed from the cultivation area before it affects other plants. These changes may be realized by drones as it captures the images of plantation area. Changes like shape/colour of the plants will be changed if the plant is affected, which can be avoided at the earlier stage by using the drones to spray pesticides.
Figure 4.1: System Architecture for checking the ripeness level of a fruit

The system architecture clearly explains the computation for ripeness detection in mangoes, when the fruit is given as an input to the system, it initially checks for the changes in colour, size, and shape as well. Even if there are minor changes in the fruit, it's getting ripened slowly, else if there are no changes observed it's yet to be a fruit. Once if we observe changes, the next step is to determine whether it's a complete or incomplete fruit with the help of image processing algorithms.

\section{Flowchart to control drones:}

As told earlier, drones can be controlled by a pilot from the ground or it can be programmed and made autonomous. When it's controlled by a pilot, he should have a clear understanding of input controls so that drone doesn't cause any harm nor it gets damaged.

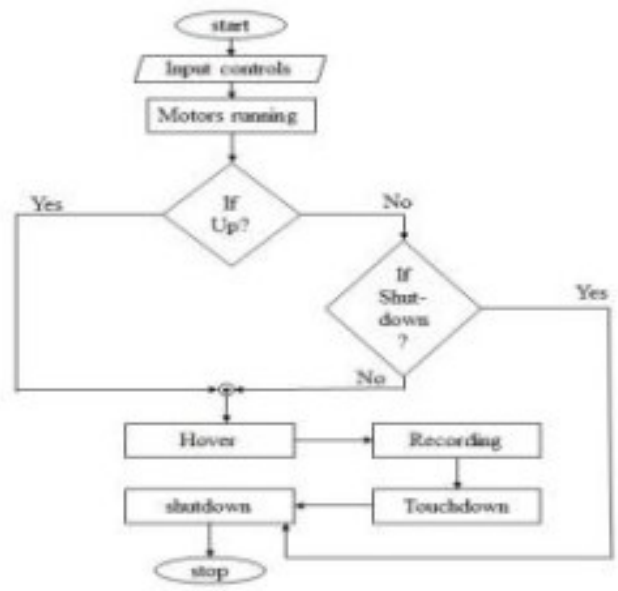

Figure 4.2: System Architecture for controlling drones 
As shown in the above diagram, one can get an idea of drone mechanism through a pilot. Its' important to know the functionalities before running this in the cultivation area.

\section{Sequence diagram for watering and spraying pesticides:}

When these activities are done with the help of a drone, lot of manual work will be eliminated or reduced which also provides precise results at a low cost.

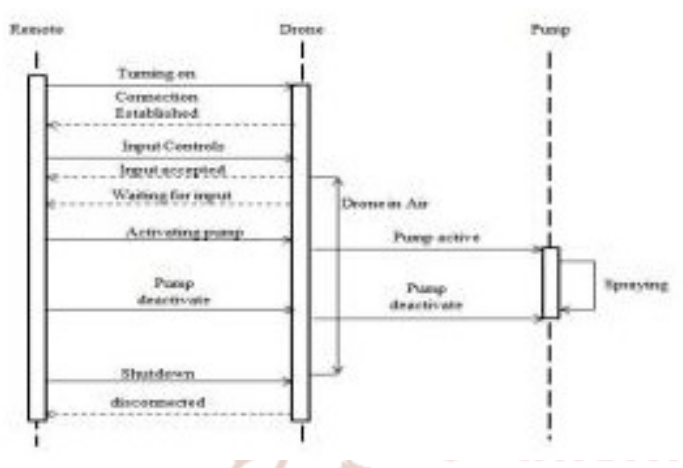

Figure 4.3: Sequence diagram for watering and spraying pesticides

When the drone is activated, the control will be in the pilot's hand who sets the threshold values at an earlier stage. Spraying pesticides using drones can reduce the man work and decrease the labour cost. Everyday watering a huge land manually isn't an easy task, so when drones come into the picture farmers can save lot of time. Just by understanding the requirements, they can set the threshold levels which inform the drone to water the plants in precise amounts. Once these activities are taken can, drones should be deactivated by the one who's controlling it.

\section{Conclusion}

Automatons can be utilized to screen, keep up and secure the harvests and the product field. By introducing the flagging gadgets at each tree or at the fringes of particular manor territory would help the automatons to separate between the handled trees or the ranch zone.

This would likewise diminish the computational power required. Utilizing image processing, the procedure of crop aging could be checked. With the assistance of shape, colour-shade, estimate and different attributes the aging and ripening procedure can be checked. This causes agriculturists to decrease the manual work and spare both cash and time. Additionally, UAVs can be utilized to screen the wellbeing of the tainted harvests, products of the soil in the manor region, it can be cured once the drone catches its picture and fitting moves can be made keeping in mind the end goal to keep it from spreading.

Diverse sort of UAVs can be utilized to capture the pictures of crops for dissecting the ripeness. Not just maturing process, even yield well-being can be checked with the assistance of drones which is practical and spares time. The work can be finished within hours by, which may take days if done physically. Automatons can be checked by a man sitting at one corner. He/she can control the automaton physically or it can likewise be mechanized with the assistance of flagging gadgets. In this way, by utilizing automatons and digital image processing, the maturing procedure of the ripening of fruits can be effortlessly analysed.

\section{References}

1) Gustavo Rezende Silva, Mauricio Cunha Escarpinati, Daniel D. Abdala, and Iuri Rezende Souza "Definition of Management Zones through Image Processing for Precision Agriculture", IEEE 2017

2) S.Delalieux, D. Raymaekers, K. Nackaerts, E. Honkavaara, J. Soukkamäki, and J. Van Den Borne "HIGH SPATIAL AND SPECTRAL REMOTE SENSING FOR DETAILED MAPPING OF POTATO PLANT PARAMETERS" 2015

3) Fintan Corrigan, "Multispectral Imaging Camera Drones In Farming Yield Big Benefits" 2016

4) Tomoya MORIBE, Hiraku OKADA, Kentaro KOBAYASHI, and Masaaki KATAYAMA, "Combination of a Wireless Sensor Network and Drone Using Infrared Thermometers for Smart Agriculture", 15th IEEE Annual Consumer Communications \& Networking Conference (CCNC) 2018

5) Geon-Hwan Kim, Imtiaz Mahmud, and You-Ze Cho, "Self-Recovery Scheme Using Neighbor Information for Multi-drone Ad Hoc Networks", 2017 
6) Sandeep Konam, "Agricultural Aid for Mango cutting (AAM)", 2015

7) Madeleine Stein, Suchet Bargoti, and James Underwood, "Image Based Mango Fruit Detection, Localisation and Yield Estimation Using Multiple View Geometry", 2016

8) Michinari Morita, Hironobu Kinjo, and Shido Sato Tansuriyavong Sulyyon Takashi Anezaki, "Autonomous Flight Drone for Infrastructure (Transmission line) Inspection (3)", Track1: Signal Processing, Computer Networks and Telecommunications, Okinawa, Japan, ICIIBMS 2017

9) Trupen Meruliya, Parth Dhameliya, Jainish Patel, Dilav Panchal, Pooja Kadam, and Sapan Naik, "Image Processing for Fruit Shape and Texture Feature Extraction - Review", 2015

10) Meenu Dadwal, and V. K. Banga, "Color Image Segmentation for Fruit Ripeness Detection: A Review", 2012

11) Rahul Pralhad Salunkhel, and Aniket Anil PatiF, "Image Processing for Mango Ripening Stage Detection: RGB and HSV method", 2015

12) C R Manjunath, PROJECT AGRO, "International Journal of research - Granthaalayah”, 2017

13) Theerapat Pobkrut, Tanthip Eamsa-ard, Teerakiat Kerdcharoen, "Sensor Drone for Aerial Odor Mapping for Agriculture and Security Services", IEEE 2016

14) Marthinus Reinecke, and Tania Prinsloo, "The influence of drone monitoring on crop health and harvest size", IEEE 2017

15) Rodrigo Filev Maia and Anh Lan Ho Tran, "Precision agriculture using remote monitoring systems in Brazil", IEEE 2017

16) Yallappa D , M. Veerangouda, Devanand Maski Vijayakumar Palled, and M. Bheemanna, "DEVELOPMENT AND EVALUATION OF DRONE MOUNTED SPRAYERFORPESTICIDE APPLICATIONS TO CROPS", IEEE 2016

17) Imre Petkovics , János Simon, Ármin Petkovics , Zlatko ýoviü, "Selection of Unmanned Aerial Vehicle for Precision Agriculture with Multicriteria Decision Making Algorithm”, IEEE 2017

18) Floriano De Rango, Nunzia Palmieri, Amilcare Francesco Santamaria, Giuseppe Potrino, "A
Simulator for UAVs Management in Agriculture Domain", IEEE 2017

19) Y.A. Pederi, H.S. Cheporniuk, "Unmanned Aerial Vehicles and New Technological Methods of Monitoring and Crop Protection in Precision Agriculture", IEEE 2015

20) David W. Matolak, "Unmanned Aerial Vehicles: Communications Challenges and Future Aerial Networking", IEEE 2015

21) Zeeshan Kaleem, Mubashir Husain Rehman, Ejaz Ahmed, Abbas Jamalipour, Joel J. P. C. Rodrigues, Hassna Moustafa, WaelGuibene,"AmateurDrone Surveillance: Applications, Architectures, Enabling Technologies, and Public Safety Issues: Part 1", IEEE Communications Magazine 2018

22) Gustavo Rezende Silva, Mauricio Cunha Escarpinati, Daniel D. Abdala, Iuri RezendeSouza,"Definitionof Management Zones through Image Processing for Precision Agriculture", IEEE 2017

23) Mónica Abarca, Carlos Saito, Javier Cerna, Renato Paredes, Francisco Cuéllar, "An Interdisciplinary Unmanned Aerial VehiclesCoursewithPractical Applications", IEEE Global Engineering Education Conference (EDUCON) 2017

24) Spoorthi.S, Dr.B.Shadaksharappa, Suraj.S, V.K.Manasa, "FREYR DRONE: PESTICIDE/FERTILIZERS SPRAYING DRONE - AN AGRICULTURAL APPROACH”, IEEE 2017

25) Jinmika Wijitdechakul, Yasushi Kiyoki, Shiori Sasaki, Chawan Koopipat, "UAV-based Multispectral Image Analysis System with Semantic Computing for Agricultural Health Conditions Monitoring and Real-time Management", International Electronics Symposium (IES) 2016 